\title{
Managing drug interactions in HIV-infected adults with comorbid illness
}

\author{
Christine A. Hughes BScPharm PharmD, Alice Tseng BScPhm PharmD, Ryan Cooper MD
}

Competing interests: For activities outside the scope of the work reported here, Christine Hughes has received personal fees from Gilead Sciences, Janssen and Merck, as well as grants from ViiV, and Alice Tseng has received grants and speaker's honoraria from AbbVie, Gilead, Merck, ViiV and Jannsen, as well as a grant from Bristol Myers Squibb. Alice Tseng has also served as an advisory board member for ViiV. No competing interests declared by Ryan Cooper.

This article has been peer reviewed.

\section{Correspondence to:} Christine A. Hughes, christine.hughes@ualberta .ca

CMAJ 2015. DOI:10.1503 /cmaj.131626
$\mathrm{W}$ ith the introduction of combination antiretroviral therapy, HIV-1 infection is now considered a treatable chronic infectious disease. Modern combination antiretroviral therapy consists of at least three antiretroviral agents and leads to virologic suppression in most patients who are able to take their medication regularly. ${ }^{1,2}$ As a result, HIV-related mortality has been greatly reduced, and the life expectancy of HIV-infected individuals is near normal. ${ }^{3}$

Currently, more than 20 approved antiretroviral drugs are available in Canada, belonging to six medication classes (Box 1). Recent advances in HIV treatment include improved tolerability of newer agents; availability of new medication classes with novel mechanisms of actions, such as the integrase inhibitors; and an increasing number of fixed-dose combination tablets, which greatly reduce the pill burden, in some cases allowing for entire HIV regimens to be provided with a single tablet administered once daily. ${ }^{2}$

As a consequence of decreased mortality and effective treatment, the demographic characteristics of the HIV-infected population have shifted: in the United States, for example, more than half of HIV-infected individuals will be 50 years of age or older by the year $2015 .{ }^{4}$ It is also now increasingly recognized that despite effective antiretroviral treatment, the incidence of cardiovascular disease, non-HIV-related cancer, and renal and hepatic impairment is greater among HIV-infected patients 50 years of age and older

\section{- Ker pOINTS}

- Older HIV-infected patients are at potentially higher risk of drug-drug interactions because of an increased incidence of comorbidities resulting in polypharmacy.

- Many antiretroviral drugs are prone to pharmacokinetic drug-drug interactions because they are substrates as well as inhibitors or inducers of several cytochrome P450 isoenzymes.

- Recommendations for management of antiretroviral drug interactions with medications commonly used in HIV-infected adults with comorbid disease are based primarily on pharmacokinetic studies in healthy volunteers and case reports or small studies in HIV-infected adults.

- Given the potential for serious consequences in older HIV-infected adults, clinicians need to screen for potential drug interactions and be aware of resources to guide management.

than among uninfected individuals of the same age. ${ }^{1,5,6}$ The premature onset of age-associated medical comorbidities is a phenomenon sometimes called "accelerated aging in HIV-infection" and may be due to effects of chronic viral infection, cumulative toxicity of long-term antiretroviral therapy and/or higher frequency of tobacco and other substance use. . $^{1,5,6}$

With a relatively high burden of comorbid conditions and diseases of aging, older HIV-infected patients are at particularly high risk of polypharmacy and drug-drug interactions. ${ }^{7}$ Relative to HIV-infected patients under 50 years of age, older HIV patients are substantially more likely to be receiving additional medications, including cardiovascular drugs, antacids or acid-suppressing agents, lipid-lowering agents, antiplatelet agents or anticoagulants, oral hypoglycemic agents and erectile dysfunction drugs. ${ }^{7,8}$ In addition, in some studies, the use of antidepressants, psychotropics and analgesics was more common among older HIV-infected patients than among HIV-infected patients younger than 50 years. ${ }^{8,9}$

As primary care physicians and other specialists become increasingly involved in the care of HIV-infected individuals, it is essential for them to recognize, prevent and manage clinically important interactions between commonly prescribed drugs and antiretroviral therapy. This review highlights potentially important pharmacokinetic interactions between antiretroviral agents and drugs that are more commonly prescribed for older HIV-infected patients than for younger patients with HIV infection. We defined clinically important pharmacokinetic drug interactions as interactions that might require dosage adjustments or drug combinations that should be avoided. We considered the following drug classes, on the basis of literature suggesting a higher frequency of use by older than by younger HIV-infected adults: acid-suppressing agents, analgesics, antiplatelet agents and anticoagulants, cardiovascular drugs, antipsychotics, statins, antidepressants and antidiabetic agents. ${ }^{7-9}$ We also considered phosphodiesterase-5 inhibitors, corticosteroids and anticonvulsants, because they are frequently used in clinical prac- 
tice and carry risks of clinically important interactions with antiretroviral agents; the results of those analyses are presented in Appendix 1 (available at www.cmaj.ca/lookup/suppl/doi:10.1503 /cmaj.131626/-/DC1). However, this review is not intended to provide comprehensive information about all potential interactions involving antiretroviral drugs.

The strategy for our literature search is summarized in Box 2. The recommendations presented in this review are based primarily on pharmacokinetic studies involving healthy volunteers and on case reports or case series for HIV-infected patients.

\section{Why are antiretroviral agents prone to pharmacokinetic drug interactions?}

Like the majority of currently prescribed medications, most antiretroviral agents are not watersoluble and therefore must be modified by the cytochrome P450 (CYP450) enzyme system in the liver before they can be excreted from the body via bile or renal filtrate. However, many antiretroviral medications, as an incidental consequence of their particular chemical structure, also exert uniquely powerful effects on the activity of the CYP450 isoenzymes, inhibiting the activity of some isoforms while inducing the activity of others. The uniquely strong effects of antiretrovirals on CYP450 isoenzymes, combined with the fact that these isoenzymes are responsible for the metabolism of many other commonly prescribed agents, leads to a number of clinically important drug interactions. Both the efficacy and the toxicity of non-HIV medications can be drastically affected by the coadministration of antiretrovirals. ${ }^{2,10}$

It is also important for HIV care providers to appreciate the bidirectional nature of HIV drugdrug interactions. Many antiretroviral agents have a narrow therapeutic window. Excessively high plasma concentrations can increase toxic effects and reduce tolerability, whereas low plasma concentrations lead to reduced virologic control and emergence of drug-resistant strains of the virus. ${ }^{2,10}$ In either case, the ability to ensure long-term, durable control of HIV infection is compromised. Thus, HIV-infected patients are considered particularly vulnerable to the pharmacokinetic influences of other medications, and any drug that reduces the absorption of an HIV medication or alters its metabolism through the CYP450 system can potentially undermine the efficacy of HIV treatment.

All members of the protease inhibitor class are particularly strong inhibitors of CYP3A4, with ritonavir being the most potent. Indeed, this drug is commonly used at low doses in HIV treatment (100-200 mg/d) not for its antiviral activity but rather to take advantage of its inhibition of CYP3A4, which "boosts" the plasma concentrations of coadministered protease inhibitors and thereby allows for once-daily dosing. ${ }^{2,10}$ Ritonavir has a greater potential for drug-drug interactions than other protease inhibitors, because it also inhibits CYP2D6 in the liver and induces CYP2B6, CYP2C19, CYP2C9 and CYP1A2. ${ }^{10,11}$

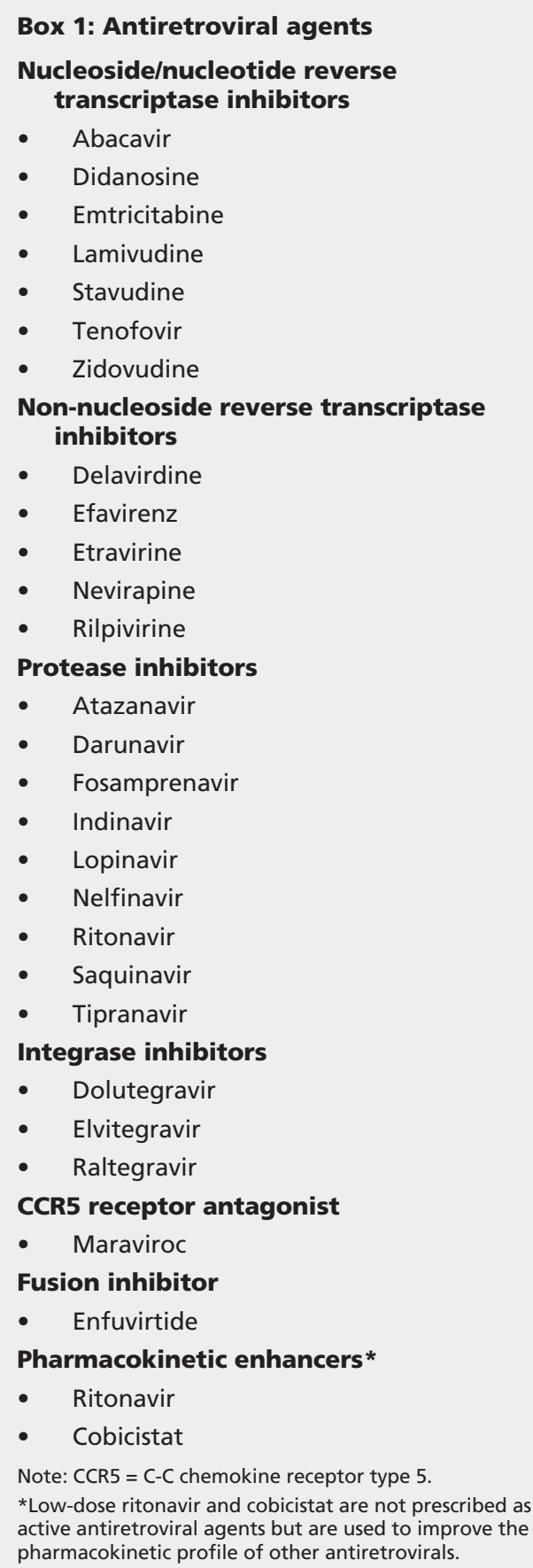


Several of the non-nucleoside reverse transcriptase inhibitors (NNRTIs) have a variety of effects on CYP450 isoenzymes. For example, efavirenz, nevirapine and etravirine are inducers of CYP3A4, whereas efavirenz and etravirine are inhibitors of CYP2C9 and CYP2C19. ${ }^{10} \mathrm{Ril}-$ pivirine does not substantially affect CYP3A4 and is therefore less commonly involved in drug-drug interactions than other members of the NNRTI class.

With regard to the integrase inhibitors, raltegravir is metabolized primarily by uridine diphosphate glucuronosyltransferase 1 family, polypeptide A1 (also known as UGT1A1) and thus has a lower potential for interactions. Elvitegravir is coformulated with cobicistat, a potent inhibitor of CYP3A4, which is used to boost concentrations of elvitegravir but is also prone to interactions with other coadministered drugs. ${ }^{12}$ Cobicistat is also an effective booster of atazanavir and darunavir, and coformulations with these agents are in development.

The nucleoside reverse transcriptase inhibitors are primarily cleared through the renal system, so pharmacokinetic interactions are much less likely to have clinical importance.

\section{What are examples of clinically important pharmacokinetic interactions with antiretrovirals?}

\section{Absorption: acid-suppressing agents}

Reduced gastric absorption of HIV medications can compromise treatment efficacy. Acid-suppressing agents used to treat gastroesophageal reflux disease and dyspepsia, such as H2-blockers or proton pump inhibitors, raise gastric $\mathrm{pH}$ and thereby reduce the

\section{Box 2: Evidence used in this review}

We performed a comprehensive search of the MEDLINE database for English-language articles (January 1998 to February 2014) using the following key search terms: "HIV" and "drug interactions" and either "calcium channel blockers" or "antiplatelet" or "anticoagulants" or "cardiovascular agents" or

"phosphodiesterase-5 inhibitors" or

"hypoglycemic" or "corticosteroids" or

"anticonvulsants" or "herbals" or "proton pump inhibitors" or "antacids" or "statins" or "analgesics" or "antidepressants" or "antipsychotics." We reviewed a total of 303 citations, and included 59 articles in our analysis. We also reviewed conference abstracts and reference lists of pertinent articles, as well as product monographs and prescribing information. The available data were limited primarily to pharmacokinetic studies involving healthy volunteers and case reports. solubility of the NNRTI rilpivirine and the protease inhibitor atazanavir, thus greatly reducing their bioavailability. Rilpivirine and atazanavir are widely used in the treatment of HIV, and this interaction is likely to be commonly encountered. ${ }^{2}$

Conversely, cationic antacids (e.g., calcium carbonate, magnesium hydroxide, aluminum sulphate) form insoluble complexes with some members of the integrase inhibitor class (e.g., elvitegravir, dolutegravir) and can substantially reduce gastric absorption of these HIV drugs. ${ }^{10}$

Several small studies involving both HIVinfected patients and healthy volunteers have evaluated the effect of the H2-receptor antagonist famotidine on serum concentrations of atazanavir. ${ }^{13-15}$ Higher doses of famotidine and its administration either 2 hours before or within 4 hours after the atazanavir dose appeared to have a greater effect on the area under the concentrationtime curve and the minimum concentration of atazanavir. ${ }^{14}$ Increasing the dose of atazanavir from 300 to $400 \mathrm{mg}$ daily (in addition to ritonavir $100 \mathrm{mg}$ daily) may compensate for this interaction. ${ }^{15}$ Famotidine also significantly reduced rilpivirine concentrations when administered 2 hours before rilpivirine. ${ }^{16}$ However, administering famotidine either 4 or 12 hours after rilpivirine did not appear to have much effect. ${ }^{16}$

Pharmacokinetic studies of proton pump inhibitors coadministered with atazanavir in healthy people have shown dramatic decreases in area under the curve and minimum concentration of atazanavir. ${ }^{17-22}$ Case series and small studies attempting to evaluate the importance of the interaction between atazanavir and proton pump inhibitors in terms of clinical outcomes (e.g., changes in viral load) have yielded inconsistent results. ${ }^{23-25}$ Nonetheless, most HIV care providers would prefer to switch to another protease inhibitor for patients requiring prolonged gastric acid suppression. Proton pump inhibitors should also be avoided in patients receiving rilpivirine, as omeprazole $20 \mathrm{mg}$ daily reduced the area under the curve and minimum concentration of rilpivirine by about $40 \% .{ }^{26}$

Although pharmacokinetic data are not available, administration of antacids at the same time as atazanavir or rilpivirine is expected to affect antiretroviral absorption because of altered gastric $\mathrm{pH}$. Separating administration times is therefore recommended. ${ }^{26,27}$

The absorption of integrase inhibitors (dolutegravir, elvitegravir and raltegravir) is substantially reduced, as a result of binding, when these drugs are coadministered with polyvalent cations, including antacids, that contain aluminum, magnesium hydroxide or calcium carbonate..$^{28-30}$ Separating administration times by at least 
2 hours has been shown to compensate for this interaction. ${ }^{28,29}$ Table 1 summarizes these interactions and possible management strategies.

\section{Metabolism: CYP3A4}

\section{Analgesics}

Interactions between antiretrovirals and methadone have been well described, ${ }^{31}$ but data regarding interactions with other opioid agonists commonly used for pain management are limited. Oxycodone and fentanyl are both metabolized by CYP3A4. Pharmacokinetic studies in healthy volunteers have shown a 2.6to 3-fold increase in oxycodone concentrations when administered with some protease inhibitors. ${ }^{32}$ The self-reported effects of oxycodone were also increased in that study. ${ }^{32}$ Similarly, ritonavir can increase the area under the curve for fentanyl, and fentanyl toxicity resulting in death has been reported following initiation of lopinavir-ritonavir as part of HIV postexposure prophylaxis. ${ }^{33,34}$ Thus, if cobicistat or a protease inhibitor is initiated in patients who are taking oxycodone or fentanyl, close monitoring and pre-emptive dose reductions may be required to avoid potential opioid-related adverse effects.

\section{Antiplatelet agents and novel oral anticoagulants}

The novel oral anticoagulants rivaroxaban and apixaban are metabolized by CYP3A4. Concentrations of these agents may be increased by protease inhibitors and cobicistat and may be decreased by NNRTIs, resulting in an increased risk of bleeding events or reduced efficacy, respectively. ${ }^{35}$ Dabigatran is cleared primarily through the renal system and thus is less likely to interact with antiretroviral agents, which gives the clinician greater flexibility in choosing an HIV regimen..$^{35}$

Table 1: Mechanisms of pharmacokinetic drug interactions between antiretrovirals and medications commonly used in older adults

\begin{tabular}{|c|c|c|c|}
\hline Mechanism & $\begin{array}{c}\text { Relevant } \\
\text { antiretrovirals }\end{array}$ & Coadministered agents & Possible management strategy \\
\hline \multicolumn{4}{|l|}{ Absorption } \\
\hline \multirow{2}{*}{ Gastric acidity } & \multirow{2}{*}{$\begin{array}{l}\text { - Atazanavir } \\
\text { - Rilpivirine }\end{array}$} & H2-receptor antagonists & $\begin{array}{l}\text { - Alter timing of administration and } \\
\text { limit dose of H2-receptor antagonist } \\
\text { - Consider increasing atazanavir dose } \\
\text { to } 400 \mathrm{mg} \text { (along with } 100 \mathrm{mg} \text { ritonavir) }\end{array}$ \\
\hline & & Proton pump inhibitors & Avoid concomitant use \\
\hline Chelation & $\begin{array}{l}\text { - Dolutegravir } \\
\text { - Elvitegravir } \\
\text { - Raltegravir }\end{array}$ & Antacids & $\begin{array}{l}\text { Separate administration times by } \\
\text { at least } 2 h^{28-30}\end{array}$ \\
\hline
\end{tabular}


The antiplatelet agent ticagrelor is also a CYP3A4 substrate, whereas prasugrel is bioactivated by CYP3A4 to its active metabolite. In a study involving healthy volunteers, inhibition of CYP3A4 by ritonavir reduced the area under the curve for the active metabolite of prasugrel by $40 \% .{ }^{36}$ For patients receiving protease inhibitors or cobicistat, use of clopidogrel may be preferable, as a clinically important interaction with the latter would not be expected. However, for HIV-infected patients receiving the NNRTI efavirenz or etravirine, prasugrel or ticagrelor may be a safer option because of a lower risk of interactions. Clopidogrel is bioactivated primarily by CYP2C19; efavirenz and etravirine can inhibit this isoenzyme, which could reduce the efficacy of clopidogrel. On the basis of the CYP2C19 inhibitory effects of efavirenz observed in an in vitro study, efavirenz was predicted to reduce the area under the curve for the active metabolite of clopidogrel in vivo by up to $29 \%$. $^{37}$

\section{Calcium-channel blockers}

Most currently used calcium-channel blockers (e.g., amlodipine, diltiazem, felodipine, nifedipine and verapamil) are metabolized by CYP3A4. Data are limited, but pharmacokinetic studies in healthy volunteers have shown that ritonavir-boosted indinavir significantly increased exposure to amlodipine $(90 \%$ increase in area under the curve) and diltiazem (27\% increase in area under the curve) ${ }^{38}$ Similarly, atazanavir increased diltiazem concentrations and area under the curve about two-fold in studies involving healthy volunteers. ${ }^{27}$ Serious adverse effects (e.g., hypotension, edema, bradycardia) have been described in case reports of patients stabilized on calciumchannel blocker therapy who were initiated on protease inhibitors. ${ }^{39,40}$ Conversely, pharmacokinetic studies have shown that efavirenz decreased diltiazem concentrations $(69 \%$ decrease in area under the curve) ${ }^{41}$ Thus, adjustment of the calcium-channel blocker dose may be necessary when protease inhibitor, cobicistat or NNRTI therapy is initiated.

\section{Psychotropic agents}

No pharmacokinetic data are available, but antipsychotics such as aripiprazole, quetiapine and ziprasidone are CYP3A4 substrates, and dose adjustments may therefore be required when these drugs are given with protease inhibitors, cobicistat or NNRTIs. ${ }^{42}$ Reports of increased adverse effects due to quetiapine ${ }^{43,44}$ and aripiprazole $^{45}$ in patients receiving protease inhibitors have been published.

\section{Statins}

Lipid disorders are frequently observed in older HIV-infected individuals, in part because of the dyslipidemic effects of some antiretroviral agents. Most statins are extensively metabolized by the CYP450 enzyme system in the liver, although the degree to which this occurs is variable. For example, lovastatin and simvastatin are lipophilic molecules that depend to a greater extent on CYP3A4 metabolism for clearance than do less lipophilic drugs. As a result, greater increases in serum concentrations of these statins occur when they are coadministered with potent CYP3A4 inhibitors such as protease inhibitors or cobicistat. ${ }^{46,47}$ As an example, a 31-fold increase in the area under the curve for simvastatin was seen when this statin was coadministered with ritonavirboosted saquinavir in healthy volunteers. ${ }^{48}$ Although drug interaction data are not available for newer protease inhibitors or cobicistat, both lovastatin and simvastatin are contraindicated for patients receiving these agents. ${ }^{2,49}$ This contraindication is further supported by small studies and case reports suggesting a greater risk of liver and muscle toxicity when lovastatin and simvastatin were coadministered with protease inhibitors. ${ }^{50}$ Conversely, atorvastatin and rosuvastatin are less lipophilic and less dependent on CYP3A4, and smaller increases in the area under the curve have been seen when these statins are combined with protease inhibitors. ${ }^{47,51}$ Atorvastatin and rosuvastatin can be safely used in HIV-infected patients who are taking protease inhibitors or cobicistat but should be administered at the lowest possible doses. ${ }^{2,49,51-56}$

Efavirenz has been shown to reduce the area under the curve for atorvastatin, simvastatin and pravastatin by about $40 \%-60 \%$ in healthy adult volunteers. ${ }^{57}$ Etravirine reduced the area under the curve for atorvastatin by $37 \%$ in pharmacokinetic studies ${ }^{58}$ whereas rilpivirine did not appear to significantly affect this variable. ${ }^{26}$ When a statin is used with NNRTIs, the statin dose may require adjustment according to lipid response; however, the dose should not exceed the maximum daily recommended dose. $^{2}$

\section{Metabolism: other CYP450 isoenzymes}

The majority of antiretroviral drug interactions involve CYP3A4, but agents metabolized through other CYP450 isoenzymes, including CYP2B6, CYP2D6, CYP2C8, CYP2C19 and CYP2C9, may also interact with antiretrovirals. Such medications include antidepressants, oral hypoglycemic agents and warfarin. 


\section{Antidepressants}

Most antidepressants, including tricyclic antidepressants, selective serotonin reuptake inhibitors, bupropion and trazodone, are substrates of CYP2D6, CYP2B6, CYP2C19 or, to a lesser extent, CYP3A4. It is therefore possible that the use of protease inhibitors or NNRTIs may increase or decrease concentrations of antidepressants, which makes close monitoring essential, with dose adjustments based on the patient's response or the occurrence of adverse effects. Pharmacokinetic data are limited with respect to interactions with antiretrovirals; however, ritonavir and other boosted protease inhibitors have been shown to reduce the area under the curve for bupropion and sertraline by up to $50 \%-60 \%$ through induction of CYP2B6. ${ }^{59-61}$ The boosted protease inhibitors fosamprenavir and darunavir have also been shown, in studies of healthy volunteers, to reduce the area under the curve for paroxetine by about $40 \%-60 \%$; however, the mechanism for this interaction is unclear, as paroxetine is primarily a substrate of CYP2D6. ${ }^{61,62}$ Serotonin syndrome was reported in a case series of patients receiving fluoxetine (a CYP2D6 substrate) in conjunction with high-dose (200-600 mg twice daily) ritonavir-based combination antiretroviral therapy ${ }^{63}$ High-dose ritonavir has also been shown to increase the area under the curve for desipramine by $145 \%$ and to increase the maximum concentration of trazodone by $33 \% .^{64,65}$ The significance of interactions with lower (boosting) doses of ritonavir is unclear, but patients starting antidepressant therapy should be carefully monitored for adverse effects, and the dose should be adjusted according to patients' response (without exceeding the maximum recommended dose).

\section{Oral hypoglycemic agents}

Diabetes mellitus is increasingly seen in HIVinfected individuals, especially as this population ages. ${ }^{6}$ Furthermore, some antiretroviral agents may impair glucose tolerance or even lead to hyperglycemia. ${ }^{2}$

Certain classes of oral hypoglycemic agents are metabolized via the CYP450 system and may be susceptible to interactions with antiretrovirals, although data are very limited ${ }^{66}$ Sulfonylureas are CYP2C9 substrates, and their concentrations may be affected by the NNRTIs etravirine and efavirenz (CYP2C9 inhibitors), the protease inhibitor ritonavir (a CYP2C9 inducer) and the integrase inhibitor elvitegravir (a CYP2C9 inducer). Repaglinide and nateglinide are substrates of CYP3A4, CYP2C8 and CYP2C9, and coadministration of protease inhibitors, elvitegravir-cobicistat and NNRTIs may increase or decrease exposure to these agents. ${ }^{66}$ Rosiglitazone, a thiazolidinedione, is a CYP2C8 substrate, whereas pioglitazone is metabolized by both CYP2C8 and CYP3A4. In an open-label, crossover study in healthy people, coadministration of atazanavir $400 \mathrm{mg}$ daily with rosiglitazone $4 \mathrm{mg}$ daily resulted in a $35 \%$ increase in area under the curve for rosiglitazone, whereas coadministration of atazanavir $300 \mathrm{mg} /$ ritonavir $100 \mathrm{mg}$ daily with rosiglitazone resulted in a $17 \%$ decrease in rosiglitazone exposure. ${ }^{67}$ The effect of NNRTI coadministration on thiazolidinedione pharmacokinetics has not been studied. Nevertheless, these classes should be coadministered with caution, as NNRTIs have inducing and/or inhibiting properties and may also affect thiazolidinedione exposure.

\section{Warfarin}

Warfarin is a racemic mixture: the CYP2C9 isoenzyme is primarily responsible for metabolism of the more potent $S$-warfarin, whereas $R$-warfarin is metabolized by CYP1A2 and CYP3A4. A pharmacokinetic study in healthy volunteers showed a $21 \%$ decrease in the area under the curve for $S$-warfarin when combined with darunavir-ritonavir. ${ }^{68}$ Numerous case reports have shown the need to monitor international normalized ratio closely when initiating protease inhibitor therapy, with substantial increases in the warfarin dose often being needed. ${ }^{69-73}$ The effects of NNRTIs, including efavirenz, are less clear. One case described the need for an increase in warfarin dose with efavirenz, ${ }^{69}$ but another reported a decrease in warfarin dose after initiation of the same NNRTI. ${ }^{72}$

\section{How can drug interactions be identified?}

There are a number of ways to improve the identification of drug interactions, including accurate recording of patients' medication therapy, computerized systems for electronic records, support from pharmacists and use of available antiretroviral drug interaction websites and other general drug interaction tools. In a retrospective study of outpatient visits to an HIV clinic, clinically important interactions were noted for $27 \%$ of 159 patients, ${ }^{74}$ but only $36 \%$ of these interactions were identified by the physician. Box 3 lists drug interaction websites for antiretrovirals that have been ranked highly for content and usefulness (reliability, accessibility, navigation). ${ }^{75}$

\section{Conclusion}

Combination antiretroviral therapy has reduced mortality from AIDS-related diseases; however, 


\section{Box 3: Useful websites for information about interactions involving antiretroviral drugs $^{75}$}

Immunodeficiency Clinic (Toronto)

www.hivclinic.ca

Antiretroviral drug interaction tables

University of Liverpool

www.hiv-druginteractions.org

Searchable drug interaction website;

downloadable app available

HIV InSite, University of California,

San Francisco

http://hivinsite.ucsf.edu/insite?page $=$ ar-00-02

Searchable drug interaction website

the management of the aging HIV-infected population presents new challenges. Antiretroviral agents, especially protease inhibitors, NNRTIs and cobicistat-boosted elvitegravir, are prone to bidirectional drug-drug interactions due to their metabolism through CYP3A4 and their potent influence on the activity of a wide range of CYP450 isoenzymes. Clinicians should routinely screen for important drug-drug interactions using available online resources before initiating new medications. Routine communication among health care professionals caring for HIVinfected patients is imperative to avoid unnecessary toxic effects and/or therapeutic failure.

\section{References}

1. Deeks SG, Phillips AN. HIV infection, antiretroviral treatment, ageing, and non-AIDS related morbidity. BMJ 2009;338:a3172.

2. Panel on Antiretroviral Guidelines for Adults and Adolescents. Guidelines for the use of antiretroviral agents in HIV-1-infected adults and adolescents. Rockville (MD): Department of Health and Human Services, AIDSinfo; updated 2014 May 1. Available: http://aidsinfo.nih.gov/guidelines/html/1/adult-and-adolescent -treatment-guidelines/0 (accessed 2014 Sept. 8).

3. Bhaskaran K, Hamouda O, Sannes M, et al. Changes in the risk of death after HIV seroconversion compared with mortality in the general population. JAMA 2008;300:51-9.

4. Effros RB, Fletcher CV, Gebo K, et al. Aging and infectious diseases: workshop on HIV infection and aging: what is known and future research directions. Clin Infect Dis 2008;47:542-53.

5. Goulet JL, Fultz SL, Rimland D, et al. Do patterns of comorbidity vary by HIV status, age, and HIV severity? Clin Infect Dis 2007;45:1593-601.

6. Nkhoma E, Vannappagari V, Mynepalli L, et al. Comorbidities and coinfections among HIV patients 50 years and older [abstract O_16]. First International Workshop on HIV \& Aging; 2010 Oct. 4-5; Baltimore.

7. Marzolini C, Back D, Weber R, et al. Ageing with HIV: medication use and risk for potential drug-drug interactions. $J$ Antimicrob Chemother 2011;66:2107-11.

8. Tseng A, Szadkowski L, Walmsley S, et al. Association of age with polypharmacy and risk of drug interactions with antiretroviral medications in HIV-positive patients. Ann Pharmacother 2013;47:1429-39.

9. Koram N, Vannappargari V, Sampson T, et al. Comorbidity prevalence and its influence on non-ARV comedication burden among HIV positive patients [abstract 323]. IDWeek 2013 [conference]; 2013 Oct. 2-6; San Francisco.

10. Rathbun RC, Liedtke MD. Antiretroviral drug interactions: overview of interactions involving new and investigational agents and the role of therapeutic drug monitoring for management. Pharmaceutics 2011;3:745-81.

11. Foisy MM, Yakiwchuk EM, Hughes CA. Induction effects of ritonavir: implications for drug interactions. Ann Pharmacother 2008;42:1048-59.

12. Stribild (elvitegravir, cobicistat, emtricitabine, tenofovir diso- proxil fumarate) [product monograph]. Mississauga (ON): Gilead Sciences Inc.; 2014

13. Agarwala S, Eley T, Vilegas C, et al. Pharmacokinetic effect of famotidine on atazanavir with or without ritonavir in healthy subjects [abstract 11]. 6th International Workshop on Clinical Pharmacology of HIV Therapy; 2005 Apr. 28-30; Quebec City.

14. Wang X, Boffito M, Zhang J, et al. Effects of the H2-receptor antagonist famotidine on the pharmacokinetics of atazanavirritonavir with or without tenofovir in HIV-infected patients. AIDS Patient Care STDS 2011;25:509-15.

15. Chung E, Zhu L, Sims K, et al. An increase in atazanavir to $400 \mathrm{mg}$ mitigates the effects of famotidine when given with ritonavir and tenofovir DF in HIV-infected patients [abstract P 14]. 13th International Workshop on Clinical Pharmacology of HIV Therapy; 2012 Apr. 16-18; Barcelona, Spain.

16. Van Heeswijk RP, Hoetelmans RM, Kestens D, et al. The pharmacokinetic $(\mathrm{PK})$ interaction between famotidine and TMC278, a next generation non-nucleoside reverse transcriptase inhibitor (NNRTI), in HIV-negative volunteers [abstract TUPDB01]. 4th International AIDS Society Conference on HIV Pathogenesis, Treatment and Prevention; 2007 July 22-25; Sydney, Australia.

17. Agarwala S, Gray K, Eley T, et al. Pharmacokinetic interaction between atazanavir and omeprazole in healthy subjects [poster WePe3.3C08]. 3rd International AIDS Society Conference on HIV Pathogenesis and Treatment; 2005 July 24-27; Rio de Janeiro, Brazil.

18. Agarwala S, Gray K, Wang Y, et al. Pharmacokinetic effect of omeprazole on atazanavir with ritonavir in healthy subjects [abstract 658]. 12th Conference on Retroviruses and Opportunistic Infections; 2005 Feb. 22-25; Boston.

19. Tomilo DL, Smith PF, Ogundele AB, et al. The effect of lansoprazole acid suppression on the pharmacokinetics of atazanavir in healthy volunteers [abstract A-1192]. 45th Interscience Conference on Antimicrobial Agents and Chemotherapy; 2005 Dec. 16-19; Washington.

20. Luber A, Brower R, Peloquin CA, et al. Steady-state pharmacokinetics of QD fosamprenavir/ritonavir and atazanavir/ritonavir alone and in combination with $20 \mathrm{mg}$ QD of omeprazole in healthy volunteers [abstract 36]. 7th International Workshop on Clinical Pharmacology of HIV Therapy; 2006 Apr. 20-22; Lisbon, Portugal.

21. Luber AD, Brower R, Kim D, et al. Steady-state pharmacokinetics of once-daily fosamprenavir/ritonavir and atazanavir/ ritonavir alone and in combination with $20 \mathrm{mg}$ omeprazole in healthy volunteers. HIV Med 2007;8:457-64.

22. Zhu L, Persson A, Mahnke L, et al. Effect of low-dose omeprazole ( $20 \mathrm{mg}$ daily) on the pharmacokinetics of multiple-dose atazanavir with ritonavir in healthy subjects. J Clin Pharmacol 2011;51:368-77.

23. Antoniou T, Yoong D, Beique L, et al. Impact of acid-suppressive therapy on virologic response to atazanavir-based regimens in antiretroviral-experienced patients: a case series. J Acquir Immune Defic Syndr 2005;39:126-8.

24. Kosel BW, Storey SS, Collier AC. Lack of interaction between atazanavir and lansoprazole. AIDS 2005;19:637-8.

25. Poirier J, Guiard-Schmid J, Bonnard P, et al. Proton pump inhibitors do not decrease atazanavir trough plasma concentrations in HIV-infected patients treated with ritonavir boosted atazanavir regimen (300/100 mg qd) [abstract H-1895]. 45th Interscience Conference on Antimicrobial Agents and Chemotherapy; 2005 Dec. 16-19; Washington.

26. Edurant (rilpivirine) [product monograph]. Toronto: Janssen Inc.; 2013.

27. Reyataz (atazanavir) [product monograph]. Montréal: BristolMyers Squibb Canada; 2013.

28. Patel P, Song I, Borland J, et al. Pharmacokinetics of the HIV integrase inhibitor S/GSK1349572 co-administered with acidreducing agents and multivitamins in healthy volunteers. $J$ Antimicrob Chemother 2011;66:1567-72.

29. Ramanathan S, Mathias A, Wei X, et al. Pharmacokinetics of once-daily boosted elvitegravir when administered in combination with acid-reducing agents. J Acquir Immune Defic Syndr 2013;64:45-50.

30. Kiser JJ, Bumpass JB, Meditz AL, et al. Effect of antacids on the pharmacokinetics of raltegravir in human immunodeficiency virus-seronegative volunteers. Antimicrob Agents Chemother 2010;54:4999-5003.

31. Bruce RD, Moody DE, Altice FL, et al. A review of pharmacological interactions between HIV or hepatitis $\mathrm{C}$ virus medications and opioid agonist therapy: implications and management for clinical practice. Expert Rev Clin Pharmacol 2013;6:249-69.

32. Nieminen TH, Hagelberg NM, Saari TI, et al. Oxycodone concentrations are greatly increased by the concomitant use of ritonavir or lopinavir/ritonavir. Eur J Clin Pharmacol 2010; 66:977-85. 
33. Olkkola KT, Palkama VJ, Neuvonen PJ. Ritonavir's role in reducing fentanyl clearance and prolonging its half-life. Anesthesiology 1999;91:681-5.

34. Cheng R, Greenall J, Koczmara C, et al. HIV postexposure prophylaxis and the need for drug interaction screening. Can J Hosp Pharm 2008;61:439-40.

35. Egan G, Hughes CA, Ackman ML. Drug interactions between antiplatelet or novel oral anticoagulant medications and antiretroviral drugs. Ann Pharmacother 2014:48:734-40.

36. Ancrenaz V. Pharmacokinetic interaction between prasugrel and ritonavir in healthy volunteers. Basic Clin Pharmacol Toxicol 2013;112:132-7.

37. Xu C, Desta Z. In vitro analysis and quantitative prediction of efavirenz inhibition on eight cytochrome enzymes. Drug Metab Pharmacokinet 2013;28:362-71.

38. Glesby MJ, Aberg JA, Kendall MA, et al. Pharmacokinetic interactions between indinavir plus ritonavir and calcium channel blockers. Clin Pharmacol Ther 2005;78:143-53.

39. Puech R, Gagnieu MC, Planus C, et al. Extreme bradycardia due to multiple drug-drug interactions in a patient with HIV post-exposure prophylaxis containing lopinavir-ritonavir. $\mathrm{Br} \mathrm{J}$ Clin Pharmacol 2011;71:621-3.

40. Izzedine H, Launay-Vacher V, Deray G, et al. Nelfinavir and felodipine: a cytochrome P450 3A4-mediated drug interaction. Clin Pharmacol Ther 2004;75:362-3.

41. Sustiva (efavirenz) [product monograph]. Montréal: BristolMyers Squibb Canada; 2008.

42. Hill L, Lee KC. Pharmacotherapy considerations in patients with HIV and psychiatric disorders: focus on antidepressants and antipsychotics. Ann Pharmacother 2013;47:75-89.

43. Pollack TM, McCoy C, Stead W. Clinically significant adverse events from a drug interaction between quetiapine and atazanavir-ritonavir in two patients. Pharmacotherapy 2009;29:1386-91.

44. Hantson P, Di Fazio V, Wallemacq P. Toxicokinetic interaction between quetiapine and antiretroviral therapy following quetiapine overdose. Drug Metab Lett 2010;4:7-8.

45. Aung GL, O'Brien JG, Tien PG, et al. Increased aripiprazole concentrations in an HIV-positive male concurrently taking duloxetine, darunavir, and ritonavir. Ann Pharmacother 2010;44:1850-4.

46. Chauvin B, Drouot S, Barrail-Tran A, et al. Drug-drug interactions between HMG-CoA reductase inhibitors (statins) and antiviral protease inhibitors. Clin Pharmacokinet 2013;52:815-31.

47. Neuvonen PJ, Niemi M, Backman JT. Drug interactions with lipid-lowering drugs: mechanisms and clinical relevance. Clin Pharmacol Ther 2006;80:565-81.

48. Fichtenbaum CJ, Gerber JG, Rosenkranz SL, et al. Pharmacokinetic interactions between protease inhibitors and statins in HIV seronegative volunteers: ACTG Study A5047. AIDS 2002; 16:569-77.

49. FDA drug safety communication: interactions between certain HIV or hepatitis C drugs and cholesterol-lowering statin drugs can increase the risk of muscle injury. Silver Spring (MD): US Food and Drug Administration; 2013. Available: www.fda.gov /Drugs/DrugSafety/ucm293877.htm\#sa (accessed 2014 Apr. 7).

50. Ahmed MH, Al-Atta Y, Hamad MA. The safety and effectiveness of statins as treatment for HIV-dyslipidemia: the evidence so far and the future challenges. Expert Opin Pharmacother 2012;13:1901-9.

51. Carr RA, Andre RJ, Bertz RJ, et al. Concomitant administration of ABT 378/ritonavir results in clinically important pharmacokinetic interaction with atorvastatin but not pravastatin [abstract 334]. 40th Interscience Conference on Antimicrobial Agents and Chemotherapy; 2000 Sept. 17-20; Toronto.

52. Hoetelmans R, Lasure A, Koester A, et al. The effect of TMC114, a potent next-generation HIV protease inhibitor, with low-dose ritonavir on atorvastatin pharmacokinetics [poster H-865]. 44th Interscience Conference on Antimicrobial Agents and Chemotherapy; 2004 Oct. 30-Nov. 2; Washington.

53. Busti AJ, Bain AM, Hall RG, et al. Effects of atazanavir/ ritonavir or fosamprenavir/ritonavir on the pharmacokinetics of rosuvastatin. J Cardiovasc Pharmacol 2008;51:605-10.

54. Samineni D, Desai PB, Sallans L, et al. Steady state pharmacokinetic interactions of darunavir/ritonavir with lipid-lowering agent rosuvastatin. J Clin Pharmacol 2012;52:922-31

55. Kiser JJ, Gerber JG, Perdhomme JA, et al. Drug drug interaction between lopinavir/ritonavir and rosuvastatin in healthy volunteers. J Acquir Immune Defic Syndr 2008;47:570-8.

56. Ramanathan S, Wang H, Stondell T, et al. Pharmacokinetics and drug interaction profile of cobicistat boosted-elvitegravir with atazanavir, rosuvastatin or rifabutin [abstract O_03]. 13th International Workshop on Clinical Pharmacology of HIV Therapy; 2012 Apr. 16-18; Barcelona, Spain.
57. Gerber JG, Rosenkranz SL, Fichtenbaum CJ, et al. Effect of efavirenz on the pharmacokinetics of simvastatin, atorvastatin, and pravastatin: results of AIDS Clinical Trials Group 5108 Study. J Acquir Immune Defic Syndr 2005;39:307-12.

58. Intelence (etravirine) [product monograph]. Toronto: Janssen Inc.; 2013.

59. Park J, Vousden M, Brittain C, et al. Dose-related reduction in bupropion plasma concentrations by ritonavir. J Clin Pharmacol 2010;50:1180-7.

60. Hogeland GW, Swindells S, McNabb JC, et al. Lopinavir/ ritonavir reduces bupropion plasma concentrations in healthy subjects. Clin Pharmacol Ther 2007;81:69-75.

61. Sekar V, De Paepe E, De Marez T, et al. Pharmacokinetic interaction between darunavir (TMC 114), a new protease inhibitor, and the selective serotonin reuptake inhibitors (SSRIs), paroxetine and sertraline [abstract P295]. 8th International Congress on Drug Therapy in HIV Infection; 2006 Nov. 12-16; Glasgow, Scotland.

62. van der Lee MJ, Blenke AA, Rongen GA, et al. Interaction study of the combined use of paroxetine and fosamprenavirritonavir in healthy subjects. Antimicrob Agents Chemother 2007;51:4098-104.

63. DeSilva KE, LeFlore DB, Marston BJ, et al. Serotonin syndrome in HIV-infected individuals receiving antiretroviral therapy and fluoxetine. AIDS 2001; $15: 1281-5$.

64. Bertz RJ, Cao G, Cavanaugh JH, et al. Effect of ritonavir on the pharmacokinetics of desipramine [abstract]. 11th International Conference on AIDS; 1996 July 7-12; Vancouver.

65. Norvir (ritonavir) [product monograph]. Saint-Laurent (QC): AbbVie Corporation; 2012.

66. Tornio A, Niemi M, Neuvonen PJ, et al. Drug interactions with oral antidiabetic agents: pharmacokinetic mechanisms and clinical implications. Trends Pharmacol Sci 2012;33:312-22.

67. Sevinsky H, Eley T, Yones C, et al. Effect of atazanavir with and without ritonavir on the pharmacokinetics of the CYP2C8 probe rosiglitazone in healthy subjects [abstract O5]. 9th International Workshop on Clinical Pharmacology of HIV Therapy; 2008 Apr. 7-9; New Orleans.

68. Sekar V, Spinosa-Guzman S, Meyvisch P, et al. Cocktail study to investigate the in-vivo drug interaction potential of darunavir co-administered with low-dose ritonavir (DRV/r) on cytochrome P450 enzymes 2D6, 2C9 and 2C19 [abstract P23]. 9th International Workshop on Clinical Pharmacology of HIV Therapy; 2008 Apr. 7-9; New Orleans.

69. Welzen MEB, Van den Berk GEL, Hamers RL, et al. Interaction between antiretroviral drugs and acenocoumarol. Antivir Ther 2011;16:249-52.

70. Liedtke MD, Vanguri A, Rathbun RC. A probable interaction between warfarin and the antiretroviral TRIO study regimen. Ann Pharmacother 2012;46:e34.

71. Hughes CA, Freitas A, Miedzinski LJ. Interaction between lopinavir/ritonavir and warfarin. CMAJ 2007;177:357-9.

72. Bonora S, Lanzafame M, D'Avolio A, et al. Drug interactions between warfarin and efavirenz or lopinavir-ritonavir in clinical treatment. Clin Infect Dis 2008;46:146-7.

73. Fulco PP, Zingone MM, Higginson RT. Possible antiretroviral therapy-warfarin drug interaction. Pharmacotherapy 2008; 28:945-9.

74. Evans-Jones JG, Cottle LE, Back DJ, et al. Recognition of risk for clinically significant drug interactions among HIV-infected patients receiving antiretroviral therapy. Clin Infect Dis 2010; 50:1419-21.

75. Sheehan NL, Kelly DV, Tseng AL, et al. Evaluation of HIV drug interaction websites. Ann Pharmacother 2003;37:1577-86.

Affiliations: Faculty of Pharmacy and Pharmaceutical Sciences (Hughes) and Division of Infectious Diseases, Department of Medicine (Cooper), University of Alberta, Edmonton, Alta.; Northern Alberta HIV Program (Hughes), Edmonton, Alta.; Toronto General Hospital (Tseng), Toronto, Ont.; Leslie Dan Faculty of Pharmacy (Tseng), University of Toronto, Toronto, Ont.

Contributors: Christine Hughes and Alice Tseng were involved in conducting the literature review and were the principal authors. All authors were involved in the analysis and interpretation of the literature. Ryan Cooper was involved in critical revision of the article. All authors approved the final version of the article, and all agree to be accountable for all aspects of the work in ensuring that questions related to the accuracy or integrity of any part of the work are appropriately investigated and resolved. 